\title{
Investigation of Chemical Cleaning of Supercritical Superheater Oxide Scale
}

\author{
Hongfeng $\mathrm{Li}^{1}$, Qingtang $\mathrm{Xue}^{1}$, Xinhui $\mathrm{Nie}^{1}$, and Yunfei $\mathrm{Xu}^{2, *}$ \\ ${ }^{1}$ Guodian Science and Technology Research Institute, Nanjing, Jiangsu 210023, P.R.China \\ 2 Department of Environment Science and Engineering, North China Electric Power University, No.689, Yonghua North Road, Baoding, \\ Hebei Province, 071000, P.R.China
}

\begin{abstract}
Recent years, as the development of thermal power plant, superheater oxide film caused by over temperature and tube explosion and the turbine blade erosion problem more and more serious. The research and development of chemical cleaning technology of superheater oxide film is one of the most effective ways to solve this problem. The chemical cleaning of superheater is rather difficult than that of boiler, for mainly the reason as below. First, the scale of superheater tube, differences in microstructure, scales dense and need to use higher cleaning medium concentration and longer cleaning time. Second, the materials of superheater complex, involves ferritic steel and austenitic stainless steel such as $12 \mathrm{Cr} 1 \mathrm{MoV}, \mathrm{T} 22$, T91, TP347 and SUS304. For controlling chemical cleaning process, the acid corrosion sample is employed to slow inhibitor on different metal and alloys. Also, the traditional weight-loss method to evaluate the corrosion rate of materials in chemical cleaning process is discussed to monitor the corrosion process.
\end{abstract}

\section{Background}

The thermal power generation enterprises have long been plagued by the problem of superheater oxide scale. When the scale in the superheater tube grows to a certain extent, the thermal expansion coefficient between the scale and the superheater metal matrix is different, and when the unit starts and stops or the loaded changes. During the change process, the scale will fall off due to the change of the main steam temperature, and the falling scale may block the superheater tube and cause local over-temperature burst or turbine blade erosion [13]. Chemical cleaning is usually used to improve the heat transfer efficiency of the equipment, slow down the formation and shedding of the superheater scale, reduce the long-term creep damage of the pipeline and the erosion of the turbine[4-5].

The chemical cleaning technology can effectively remove the oxide scale on the surface of the superheater tube, and avoid the superheater tube burst accident caused by the falling off of the scale during the long service period. In the "Guidelines for Chemical Cleaning of Boilers in Thermal Power Plants" (DL/T794, China), the guiding principle of "chemical cleaning" can be carried out for the chemical cleaning of superheaters, "the amount of scale exceeds $400 \mathrm{~g} / \mathrm{m}^{2}$, or when the explosion occurs due to oxide scale falling off." There are no specific rules for cleaning conditions.

\section{Thickness of oxide scale detection technology}

At present, the commonly used oxide scale detection methods include radiation detection, magnetic detection, high-frequency ultrasonic detection, etc. [6-8]. Among them, magnetic detection technology and high-frequency ultrasonic detection methods are more common. (1) The radiation detection method is based on the principle that $\mathrm{x}$-ray or $\mathrm{y}$-ray is different in intensity attenuation when irradiating the metal substrate and the oxide, similar to the chest piece for medical diagnosis, the scale is intuitive and easy to observe with low cost and operation. Limited to the field environment, and because the scale is an epitaxial film grown on a metal substrate, the resolution is low. (2) The high-frequency ultrasonic detection method is based on the principle of different density and acoustic impedance between the components of the two-phase material at the steel and oxide scale interface. The operation is convenient with highly efficient and the precision can reach $0.01 \mathrm{~mm}$, also the thickness of the inner wall of the tube can be measured. T91 tubing commonly used in superheaters can be used for scale testing. (3) The magnetic detection method is based on the different principles of ferromagnetism of austenitic steel and its inner wall oxide scale (mostly different valence oxides of iron). This method can measure the accumulation of scale in the tube with flexible and efficient, but it is only suitable for austenitic stainless steel tubes. The scale non-cutting detection technology of the superheater tube needs further study

In addition to non-destructive testing of superheater technique, the cut-tube inspection is often considered a more accurate method of obtaining oxide scale thickness. For the as discussed method to obtain the scale thickness,

* Corresponding author: lucifertan@163.com 
it is necessary to define "the peeling critical thickness of the scale" and "the peeling average thickness of the scale". The former is the minimum thickness at which scale peeling occurs. When the superheater and the reheater tubes reach the critical thickness of the scale peeling, there is a risk of the scale falling off during the shutdown process of power plant. The latter refers to the statistical average thickness of the scale of the scaleshedding unit. When the superheater and the reheater tube reach the average thickness of the scale peeling, the risk of scale peeling during the shutdown process is high.

By counting the scale peeling of superheater tubes such as $12 \mathrm{CrMoV}, \mathrm{T} 23$, T91 and TP347, the critical thickness of scale peeling of various materials was obtained, as shown in the following table. For lowchromium ferritic steel of superheater, the inner wall scale peeling tendency is relatively low, and the critical thickness is $0.22 \mathrm{~mm}$; for T91 steel, the inner wall scale peeling tendency is high, and the critical thickness of peeling is $0.16 \mathrm{~mm}$; For the stainless steel, the inner wall scale peeling tendency is greater, and the critical thickness of peeling is $0.07 \mathrm{~mm}$. When the thickness of the oxide of different materials exceeds the critical thickness, the supervision of the peeling of these materials shall be strengthened, and the chemical cleaning shall be considered to remove the scale[9].

Table.1. Critical thickness of scale peeling of various inner walls of superheater steel $(\mathrm{mm})$

\begin{tabular}{|c|c|c|}
\hline Materials & $\begin{array}{c}\text { the peeling critical } \\
\text { thickness of the } \\
\text { scale }\end{array}$ & $\begin{array}{c}\text { the peeling } \\
\text { average thickness } \\
\text { of the scale }\end{array}$ \\
\hline 12CrMoV & 0.22 & 0.30 \\
T23 & 0.17 & 0.21 \\
T91 & 0.16 & 0.21 \\
TP347 & 0.07 & 0.092 \\
\hline
\end{tabular}

\section{Research of cleaning process of the over heater and reheater}

The glycolic acid-formic acid composite organic acid cleaning process has the advantages of high descaling efficiency, low corrosion rate, low chloride ion content, easy disposal of waste liquid, and is a safe and efficient cleaning medium. According to the characteristics of the scale component, a glycolic acid-formic acid composite organic acid was used as the cleaning medium for the chemical cleaning of superheater oxide scale.

The scope of this chemical cleaning includes: part of the water supply pipeline, the economizer and its header, the water wall and its header, steam drum and down pipe.

The cleaning is carried out by using 2 4\% glycolic acid $+1 \sim 2 \%$ formic acid, adding special organic acid corrosion inhibitor and related additives in the cleaning solution, using citric acid rinsing and hydrogen peroxide passivation. The specific cleaning process parameters are as follows.

The pickling process parameters of the composite acid concentration of $4 \sim 6 \%$, the corrosion inhibitor concentration of $0.3 \sim 0.5 \%$, the concentration of the cosolvent $0.05 \sim 0.1 \%$, cleaning temperature $80 \sim 90^{\circ} \mathrm{C}$, cleaning time is expected $8 \sim 12 \mathrm{~h}$, the specific time is from the pickling curve.

The rinsing process parameter rinsing liquid is $0.2 \sim 0.5 \%$ citric acid, $\mathrm{pH} 3.5 \sim 4.0$ (ammonia water adjustment), temperature $50 \sim 70^{\circ} \mathrm{C}$ with rinsing time 2 4h.

Passivation process parameter passivation solution is rinsing liquid + ammonia water $+0.1 \sim 0.2 \%$ hydrogen peroxide, $\mathrm{pH}$ 9.5 10.0 (ammonia water adjustment), temperature $50 \sim 55^{\circ} \mathrm{C}$, passivation time $2 \sim 4 \mathrm{~h}$.

After the cleaning, the water-cooled wall was cut and examined. As a result, the deposit on the inner surface of the tube was completely cleaned, and the metal substrate was exposed. The passivation film was intact and evenly dark gray.

In this chemical cleaning, the average corrosion of the corrosion indicator sheet is $16.2 \mathrm{~g} / \mathrm{m} 2$, and the average corrosion rate is $1.01 \mathrm{~g} /(\mathrm{m} 2 \cdot \mathrm{h})$, which is much smaller than the value specified in DL/T794-2001" Guidelines for Chemical Cleaning of Thermal Power Plant Boilers". Use a two-column format, and set the spacing between the columns at $8 \mathrm{~mm}$. Do not add any page numbers.

\section{Monitoring of ferrous ions during chemical cleaning}

In boiler chemical cleaning, there are two ways to enter the iron ions in the wash solution. The medium in contact with the inner surface of the boiler metal and its surroundings forms a variety of ferrites under long-term chemical and electrochemical action. Compounds, which are intercalated in metal surface deposits (scales), are dissolved in the cleaning solution along with surface deposits during pickling. The reaction mechanism is as follows[10]:

$$
\begin{aligned}
& \mathrm{Fe}_{2} \mathrm{O}_{3}+6 \mathrm{H}^{+} \rightarrow 2 \mathrm{Fe}^{3+}+3 \mathrm{H} 2 \mathrm{O} \\
& \mathrm{Fe}_{2} \mathrm{O}_{3} \mathrm{FeO}+8 \mathrm{H}^{+} \rightarrow 2 \mathrm{Fe}^{3+}+\mathrm{Fe}^{2+}+4 \mathrm{H}_{2} \mathrm{O} \\
& \mathrm{FeOOH}+3 \mathrm{H}^{+} \rightarrow \mathrm{Fe}^{3+}+2 \mathrm{H}_{2} \mathrm{O} \\
& \mathrm{FeO}+2 \mathrm{H}^{+} \rightarrow \mathrm{Fe}^{2+}+\mathrm{H}_{2} \mathrm{O}
\end{aligned}
$$

The $\mathrm{Fe}^{3+}$ produced by the above reaction passes through the "shield" of the corrosion inhibitor, participates in the depolarizing corrosion of $\mathrm{Fe}$, and produces 1.5 times $\mathrm{Fe}^{2+}$ than $\mathrm{Fe}^{3+}$. As the concentration of $\mathrm{Fe}^{3+}$ in the cleaning solution continues to increase, this corrosion is also becoming more and more serious.

During the cleaning process, the corrosion resistance of the corrosion inhibitor is limited or insufficient, and other reasons cause the boiler metal to be inevitably corroded to varying degrees. This is mainly due to the depolarizing corrosion of $\mathrm{H}^{+}$to $\mathrm{Fe}$ :

$$
\mathrm{Fe}+2 \mathrm{H}^{+} \rightarrow \mathrm{Fe}^{2+}+\mathrm{H}_{2}
$$

In the above two pathways, the iron ions produced by the first cause mainly appear in the high oxidation state of $\mathrm{Fe}^{3+}$, which can also be explained by the formation of metal surface deposits which need to undergo a longer oxidation reaction process, while iron The valence electron configuration $3 \mathrm{~d} 64 \mathrm{~s} 2$ always has a strong tendency to transition to its outer electron $3 \mathrm{~d} 5$ half-filled stable structure. The iron ion produced by the second 
reason is generally present in the low oxidation state of $\mathrm{Fe}^{2+}$. This is advantageous for accurately determining the source and quantity of $\mathrm{Fe}^{2+}$ in the cleaning solution.

When the boiler is chemically cleaned, the acid concentration, $\mathrm{Cu}^{2+}, \mathrm{Fe}^{3+}$ and $\mathrm{Fe}^{2+}$ concentration are generally monitored during the cleaning process. When the concentration of $\mathrm{Fe}^{3+}$ exceeds a certain value, an appropriate amount of reducing agent is added to the cleaning liquid to reduce the concentration thereof, and the corrosion of the boiler metal is slowed down. Usually the $\mathrm{Fe}^{2+}$ is not given enough attention. The significance of monitoring $\mathrm{Fe}^{2+}$ is only when the cleaning liquid is used. When the concentration and the content of $\mathrm{Fe}^{2+}$ are not significantly changed, the pickling can be ended. There is no corresponding indicator monitoring requirement for the change of $\mathrm{Fe}^{2+}$ concentration, and emergency measures should be taken when the concentration change of $\mathrm{Fe}^{2+}$ is abnormal. This gives people an impression that there seems to be no direct causal relationship between the change in the concentration of $\mathrm{Fe}^{2+}$ in the cleaning solution and the success or failure of a boiler cleaning. This is a problem that needs to be deepened. It is well known that in order to investigate the degree of corrosion of boiler metal in cleaning, it is required to carry out the coupon test in synchronization, and to infer the degree of corrosion of the metal in the cleaning according to the weight loss value of the coupon after cleaning. Although this method has certain reference value, it is fundamentally incapable of guiding the safe cleaning of the boiler in a timely and correct manner, especially when there is an abnormality in the process. For small screening tests for corrosion inhibition efficiency before cleaning, sometimes the results of the test are inconsistent with the results of actual scale cleaning. This is because modern engineering technology involves many variables, sometimes due to one of the factors. Accidental omissions can lead to unpredictable results.

During the pickling process, the content of $\mathrm{Fe}^{2+}$ is gradually increased. However, if the content of $\mathrm{Fe}^{2+}$ continues to rise rapidly, the acid washing should be stopped urgently. There are two reasons for the increase of $\mathrm{Fe}^{2+}$ content. First, $\mathrm{Fe}^{2+}$ in the cleaning solution comes from the scaly. In the middle, the second is the result of the strong action of $\mathrm{H}^{+}, \mathrm{Fe}^{3+}$ and $\mathrm{H}^{+}+\mathrm{Fe}^{3+}$ on $\mathrm{Fe}$. There are two ways to deal with this situation. One method is that as long as the $\mathrm{Fe}^{3+}$ content is not seriously exceeded, it is not necessary to pay attention to $\mathrm{Fe}^{2+}$. One method is to stop cleaning, and the first method is generally used, and the $\mathrm{Fe}^{3+}$ content is used as a monitoring indicator for boiler cleaning. It has been found through practice that high content of $\mathrm{Fe}^{3+}$ is important, and sometimes low content of $\mathrm{Fe}^{3+}$ is detected because it is in a trough after a new round of $\mathrm{Fe}^{3+}$ to $\mathrm{Fe}^{2+}$ action, so it is easy to be ignored. In short, no matter where the source of corrosion comes from, its reaction products will be expressed in the form of $\mathrm{Fe}^{2+}$, and if it is developed, it will endanger the safe operation of the boiler in the future, leaving a stable situation.

\section{Treatment of cleaning waste solution}

Citric acid is the most widely used organic acid in equipment cleaning. It is mainly used to clean oxide scale on the surface of equipment with high cost. However, the treatment of citric acid waste liquid is difficult. Domestic citric acid cleaning waste liquid is usually discharged to the coal yard and incinerated with coal burning. This method is simple and easy, but may cause boiler water wall coking and burner corrosion. Therefore, its use has been restricted in recent years[11].

The pollutants in the pickling waste liquid mainly include the residual agent added during the pickling process and the copper and iron dissolved in the system. The treatment process is as follows: (1) Sampling: Sampling and analysing the acidity of the waste liquid, and calculating the amount of limestone required to neutralize the waste liquid. (2) Neutralization and aeration oxidation: the acid washing waste liquid is discharged to the waste liquid pool, the aeration device is aerated, the limestone is slowly added to the $\mathrm{pH}$ of the waste liquid is about 12, and the aeration is continued for 24 hours and then allowed to stand for more 12 hours. (3) Circulating filtration: start the waste pump, filter the waste liquid through the filter and discharge it back to the waste liquid pool. (4) Emissions: continue to filter until the indicators meet the requirements.

The main pollutant of the passivation liquid waste liquid is sodium nitrite. Since sodium nitrite will become a carcinogen nitrosamine under certain conditions, it must be converted into harmless substances. The treatment process of the passivation waste liquid is as follows: sodium hypochlorite oxidation, alkali neutralization, aeration, stirring degreasing and COD, standing, activated carbon + multi-media filter in addition to COD and colour, sampling and analysis of $\mathrm{pH}$ and TOC to pass and emission. (1) Sampling: Sampling and analysing the concentration of sodium nitrite in the waste liquid, and calculating the amount of sodium hypochlorite required. (2) Add sodium hypochlorite oxidation: Discharge the waste liquid to the waste liquid pool, slowly add sodium hypochlorite, turn on the aeration device to aerate, and mix the waste liquid with sodium hypochlorite. (3) Neutralization, aeration oxidation: slowly add limestone, adjust the $\mathrm{pH}$ of the waste liquid to $7.5-9$, continue to aerate for $24 \mathrm{~h}$ and then let stand for 12h. (4) Circulating filtration: start the waste liquid pump, filter the waste liquid through the filter and discharge it back to the waste liquid pool. (5) Emissions: continue to filter until the indicators meet the requirements and then effluent.

Rinse, that is, rinse after washing, is carried out after the end of the passivation process, which contains a small amount of passivation solution, and the treatment process is as follows: (1) Sampling: Sampling and analysing the $\mathrm{pH}$ of the waste liquid, and calculating the amount of sulfuric acid (or limestone) required accordingly. (2) Neutralization and aeration oxidation: the passivation waste liquid will be discharged to the waste liquid pool, the aeration device will be aerated, and $50 \%$ sulfuric acid (or limestone) will be slowly added until the $\mathrm{pH}$ of the waste liquid is between 7.5-9. 
Continue to aerate for 24 hours and then let stand for more 12 hours. (3) Circulating filtration: start the waste liquid pump, filter the waste liquid through the filter and discharge it back to the waste liquid pool. (4) Emissions: continue to filter until the indicators meet the requirements and then effluent.

The treated pickling waste can be discharged to the outside after meeting the standard.

\section{Conclusion}

The problem of superheater scale has long plagued thermal power generation companies. When the scale grows to a certain extent, due to the large difference in thermal expansion coefficient between the scale and the superheater metal material, the scale peeling occurs due to the change of the main steam temperature during the start-stop and load-lifting process of the unit, which greatly increases Risk of superheater squibs and turbine blade erosion caused by scale. The chemical cleaning of the superheater is mainly to slow the formation and shedding of the scale, reduce the long-term creep damage of the pipeline and the erosion of the turbine. Research on chemical cleaning technology for superheater scales has received increasing attention. Choose the appropriate cleaning agent and additives, depending on the material of the superheater. Choosing the right process conditions and evaluating and monitoring the cleaning process will help to obtain safe and reliable superheater cleaning technology.

\section{References}

1. J Y Cao, J Chen, Electr. Power, 3620 (2003)

2. E. W. Delong. Corros., Houston, NACE, (1985)

3. J. W. Horvath, H. D. Johnson. TAPPI Press : Proceedings of the 1998 TAPPI International Engineering Conference, Miami, 505 (1998)

4. Q F Jiao, B Yao, Electr. Power, 3646 (2004)

5. L G Wu, J F Shen,H Z Fan, et al. Electr. Power, 46 $46(2013)$

6. C Y Wang, P Wang, Clean. World, 221 (2011)

7. G Z Qin, Chemical Equipment and Anticorrosion, 6 29 (2000)

8. $\mathrm{C}$ W Yin, $\mathrm{H}$ Q Yang, Journal of Xi'an Technological University, 287 (2013)

9. J Y Cao, J X Song. Therm. Power Gener., 09 29(2009)

10. G H Dai, Northeast Electric Power Technology, 2 36(1998)

11. X L Zhang, Y G Wang, M X Sun,H Q Li. Corros. Sci. Prot. Technol., 24536 (2012) 